REVISIÓN

\title{
REVISIÓN: NIRS EN EL ANÁLISIS DE ALIMENTOS PARA LA NUTRICIÓN ANIMAL
}

\section{NIRS FOR ANALYZING ANIMAL NUTRITION FOOD: A REVIEW}

\author{
PhD. Astrid Rivera Rivera ${ }^{a}, \mathrm{PhD}(\mathrm{c})$. Jose Manuel Alba Madonado ${ }^{\mathrm{b}}$ \\ ${ }^{\mathrm{a}, \mathrm{b}}$ Universidad Francisco de Paula Santander, Grupo de investigación GI@DS \\ Ocaña, Vía Acolsure-Sede el Algodonal, Colombia, ariverar@ufpso.edu.co
}

Fecha de recepción: 01-05-2017

Fecha de aprobación: 22-06-2017

\begin{abstract}
Resumen: El análisis de la composición nutricional de forrajes y de los alimentos usados en nutrición animal es relevante en la toma de decisiones dentro del proceso productivo. La espectroscopia por infrarrojo cercano (NIRS) es una metodología que se basa en la quimiométrica, asociando la luz absorbida en una muestra de alimento con la composición química de la misma y con base en ello se desarrollan ecuaciones de predicción por cada componente químico del alimento. La metodología ha sido aplicada en el análisis de forrajes con resultados confiables para la predicción materia seca, proteína, carbohidratos estructurales, solubles, grasa y en leguminosas para la identificación de factores antinutricionales. Para el desarrollo de modelos de predicción por componente, se deben colectar muestras que abarquen todos los factores de variación de la composición química del alimento. Los modelos de predicción son desarrollados en tres fases: calibración, validación interna y validación externa en las cuales el modelo se evalúa de acuerdo a criterios estadísticos. El NIRS es una metodología que ha sido reconocida como confiable, de bajo costo, rápida y que durante el proceso no genera desechos químicos.
\end{abstract}

Palabras clave: Composición nutricional, quimiometría, calibración.

Abstract: Nutritional composition analysis of forage and food used in animal nutrition is relevant for making decision within the productive process. Near infrared spectroscopy (NIRS) is a methodology based on chemometrics, associating the light absorbed in a sample of food with the chemical composition and based on it, it develops prediction equations for each chemical component of the food. The methodology has been applied in the analysis of forages with reliable results for the prediction of dry matter, protein, structural carbohydrates, soluble carbohydrates, fat and in legumes for the identification of antinutritional factors. For the development of predictive models by component, samples should be collected covering all factors of variation of the chemical composition. The prediction models are developed in three steps: calibration, internal validation and external validation in which the model is evaluated 
according to statistical parameters. NIRS is a methodology that has been recognized as reliable, low cost, fast and does not generate chemical waste.

Keywords: calibration, chemometrics, chemycal composition

\section{INTRODUCCIÓN}

En la ganadería de carne y/o leche, la fuente primaria de la alimentación son los forrajes, no obstante estos presentan variaciones en cantidad y calidad asociadas a factores edafoclimáticos y/o de manejo. El análisis de la composición nutricional de forrajes y de los alimentos usados en las dietas de bovinos es importante en la toma de decisiones en el sistema productivo; sin embargo, las metodologías utilizadas demandan tiempo y alto costo para la obtención de los resultados. La espectroscopia por infrarrojo cercano (NIRS) es una metodología que se basa en la quimiométrica, que relaciona la luz absorbida en una muestra con la composición química de la misma y con base en ello se desarrollan ecuaciones de predicción ha sido aplicada en el análisis de productos de alimentos, químicos, del ambiente $\mathrm{y}$ en farmacéutica entre otros (Brogna et al. 2009). La metodología ha sido aplicada en el análisis de forrajes con resultados confiables para la predicción materia seca, proteína, carbohidratos estructurales, solubles, grasa y en leguminosas para la identificación de factores antinutricionales como taninos $y$ fenoles (Landau, Glasser, and Dvash 2006).

Dentro de las líneas de investigación del Departamento Pecuario de la FCAA de la UFPSO el desarrollo y uso de tecnologías limpias y sostenibles son parte del enfoque del programa de Zootecnia, por lo cual divulgar técnicas de laboratorio que estén acorde con los lineamientos de la institución es relevante en el desarrollo de la investigación, la extensión y la docencia. Esta revisión es adicional a los resultados del proyecto de investigación "Evaluación de la inclusión de levaduras vivas en la alimentación de vacas de doble proósito durante la fase de transición”, que apoyará en la evaluación de los resultados finales. El objetivo es describir el principio de la espectroscopia de infrarrojo cercano y su aplicación en los análisis de la composición nutricional usados en la alimentación animal.

\section{Reseña Histórica}

El origen del uso de la espectroscopia del infrarrojo cercano se remonta a mediados del siglo XVII, cuando Isaac Newton advirtió que al hacer pasar un haz de luz a través de un prisma, ésta se descomponía en los colores del espectro, y al volverla hacer pasar por otro prisma, la luz se podía recomponer dando lugar de nuevo a luz blanca. Esto llevó a Newton a concebir la luz solar como un compuesto de luz de diferentes colores, siendo este el punto de partida para el estudio de la espectroscopia (Peña, 2012). En cuanto a la región del infrarrojo cercano, el astrónomo Herschel en 1800 la estudio por primera vez, divulgando información que sirvió como base para el desarrollo de equipos de comunicación. Posteriormente, fue desarrollado el detector fotoeléctrico infrarrojo y en 1950 en el Departamento de Agricultura de los Estados Unidos (USDA), K. Norris lo usó para investigar las propiedades ópticas de la luz en materiales biológicos (Givens et al., 1997). El primer reporte de la aplicación de la espectroscopia de infrarrojo cercano 
(NIRS) para el análisis de alimentos fue realizado en soya por Ben-Gera y Norris (1968), en 1976 se llevaron a cabo los primeros análisis en forraje y posteriormente fue utilizado en la evaluación de materiales sólidos. Ha sido ampliamente usado para determinar la composición y calidad de heno, silo, granos y productos alimenticios, así como en la industria farmacéutica y en industrias para controlar el material usado en muelles de carga. En procesos biológicos ha sido usado para monitorear procesos de fermentación y reacciones químicas (D’Mello, 2000). En Colombia existen reportes del uso de NIRS para el análisis de forrajes realizados por Lascano, 2002 y en 2004 por Vásquez analizando forrajes de la sabana tropical (Sandoval-Mejía, BuesoUclés, \& Vélez-Nauer, 2008) y según reportes de Rivera, A.R. en Meeting \& City, 2016 fue usado para análisis de kikuyo en Antioquia, además ha sido aplicado en el análisis de yuca y caña de azúcar, entre otros (Jiménez, 2007).

\section{Descripción de la técnica}

La técnica se basa en la quimiométrica, la cual combina la espectroscopia, la estadística y la computación para desarrollar modelos matemáticos (Jiménez, 2007), es así que una muestra es irradiada con un haz de luz del infrarrojo cercano y la cantidad de luz absorbida es registrada para relacionarla con la presencia de grupos funcionales de las moléculas presentes en dicha muestra (Givens et al., 1997). El NIRS registra la absorción de energía en enlaces de $\mathrm{C}-\mathrm{H}, \mathrm{N}$ $\mathrm{H}$ y $\mathrm{O}-\mathrm{H}$ que se encuentran presentes en componentes orgánicos; de esta manera, cuando la luz entra en contacto con la materia, induce la absorción de energía únicamente en los enlaces que vibren con una frecuencia similar a la energía incidente. La absorción puede ser débil o fuerte conforme a la naturaleza de los enlaces químicos de los compuestos solidos o líquidos; de esta manera cada grupo funcional absorbe luz de la región NIR a una frecuencia y longitud de onda específica representado en el espectro como picos de absorción (Tabla 1) (D’Mello, 2000).

Tabla 1. Grupo funcional y la frecuencia de absorción en la región NIR

\begin{tabular}{llc}
\hline & $\begin{array}{c}\text { Grupo } \\
\text { Funcional }\end{array}$ & $\begin{array}{c}\text { Frecuencia } \\
\left(\mathbf{c m}^{-1}\right)\end{array}$ \\
\hline $\mathrm{OH}$ & Alcohol & $3580-3650$ \\
& Enlace $\mathrm{H}$ & $3210-3550$ \\
$\mathrm{NH}$ & Acido & $2500-2700$ \\
\hline $\mathrm{CH}$ & Amina & $3300-3700$ \\
& Alcano & $2850-2960$ \\
& Alqueno & $3010-3095$ \\
& Alquino & 3300 \\
$\mathrm{C} \equiv \mathrm{C}$ & Aromático & $\sim 3030$ \\
$\mathrm{C}=\mathrm{C}$ & Alquino & $2140-2260$ \\
& Alqueno & $1620-1680$ \\
$\mathrm{C}=\mathrm{O}$ & Aromático & $\sim 1600$ \\
& Aldehído & $1720-1740$ \\
& Cetona & $1675-1725$ \\
& Acido & $1700-1725$ \\
& Ester & $1720-1750$ \\
\hline $\mathrm{C} \equiv \mathrm{N}$ & Nitrilo & $2000-2300$ \\
\hline $\mathrm{NO}{ }_{2}$ & Nitro & $1500-1650$ \\
\hline Fuente: $\mathrm{D}$ 'Mello, & 2000 &
\end{tabular}

La radiación infrarroja que incide sobre una muestra sólida toma una trayectoria de acuerdo a la presencia de los grupos funcionales; así, una parte de la radiación es reflejada de la superficie de la muestra, otra proporción entra a la muestra siendo en ocasiones absorbida por ella y aquella que no es absorbida puede ser transmitida a través de la muestra y/o reflejada por esta (Figura 1) (Givens et al., 1997).

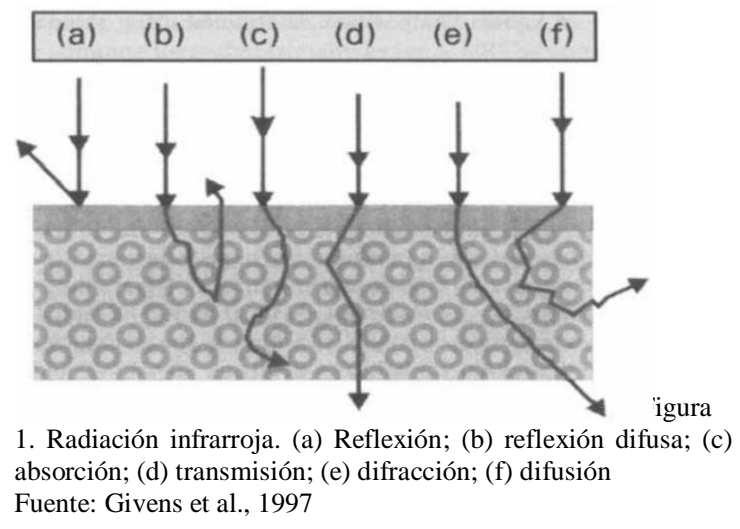


La cantidad de radiación reflejada de la muestra es cuantificada como la reflectancia de la muestra $(\mathrm{R})$ expresado como $\log (1 / \mathrm{R})$. La curva del $\log (1 / \mathrm{R})$ se compara a una curva de absorción de valores pico a ondas de longitud que corresponden a las bandas de absorción de la muestra en los grupos funcionales (Norris et al., 1976).

\section{Equipo para espectroscopia}

Los espectroscopios constan de cinco componentes básicos y pueden ser de reflectancia o de transmitancia, siendo utilizados los de reflectancia para sólidos y los de transmitancia para sustancias liquidas (Pasquini, 2003)

1. 1. Fuente de radiación: La fuente más común de radiación es la lámpara halógena con filamentos de Tungsteno con ventana de cuarzo. Se trata de una fuente de elevada intensidad de emisión y que cubre ampliamente la zona de infrarrojo cercano en el intervalo $320-2500 \mathrm{~nm}$.

2. Sistema de selección de longitud de onda: se clasifican en dos grupos dispersivos y no dispersivos. Dentro de los sistemas dispersivos se encuentran los monocromadores, estos mediante un prisma dispersa la radiación en longitudes de onda individual. En los sistemas no dispersivos, se usan filtros convencionales, los cuales limitan la radiación, permitiendo el paso de determinadas longitudes de onda.

3. Recipiente para la muestra: son cubetas dispuestas para colocar una determinada cantidad de muestra. Está ubicado entre el sistema de selección de onda y el detector. El recipiente tiene que ser transparente a la región espectral que se esté utilizando y debe ser de un material libre de ralladuras, aristas, limpio y sin burbujas cuando la muestra es líquida.

4.Detector de radiaciones o transductor: existen dos tipos de detectores, el de fotones, en el cual la luz recibida desencadena la liberación de electrones generando una corriente eléctrica y los térmicos en los cuales la luz recibida genera un aumento de temperatura, que es registrada mediante diversos sistemas. El más usado para la región de menos de $1100 \mathrm{~nm}$ es el de silicio y para la región de 1100 a 2500nm se utiliza el de sulfuro de plomo.

5. Sistema de procesamiento de la señal: realiza la lectura del detector y la registra como transmitancia, absorbancia y/o concentración que se visualiza como un espectro (Givens et al., 1997).

En los equipos para NIRS la detección de la radiación ocurre fotoeléctricamente, así la incidencia de los fotones afecta directamente el estado de los electrones en el material fotosensible de los detectores, generando un impulso eléctrico. Para minimizar la pérdida de radiación reflejada o transmitida por la muestra, los detectores son colocados cerca de la muestra a un ángulo de $45^{\circ}$. Cuando los detectores registran las ondas de luz reflejadas son transformados a datos sobre los cuales es aplicado el logaritmo como herramienta para transformar un rango amplio de datos y relacionarlo con datos más lineares. Los datos finalmente son presentados como $\log _{10} 1 / \mathrm{R}$ (Givens et al., 1997)

\section{Características de la muestra}

Las muestras que van a ser analizadas en el NIRS deben considerar todos los factores que afecten la composición química del alimento a ser analizado, ya que de ellos depende el desarrollo de los modelos de predicción precisos y exactos. Las muestras sólidas que van a ser utilizadas en equipos de reflectancia deben ser previamente secas y molidas. De esta manera, se mantienen controlan factores que puedan afectar los resultados. Los principales factores que deben ser controlados en la muestra antes de tomar espetros en el equipo NIRS son: humedad, tamaño de la partícula y temperatura, ya que en el caso de la humedad, 
los enlaces de hidrógeno y oxígeno del agua, absorben fuertemente la radiación del infrarrojo.

El método de secado de la muestra más recomendado es por liofilización, aunque debido al costo de esta metodología el más usado es el convencional en estufa (Cozzolino et al., 2005). El tamaño de la partícula debe ser homogéneo, de lo contrario absorberá mayor radiación, en la medida que la partícula sea de gran tamaño. Para controlar este factor deben ser usados molinos calibrados con cribas para 1 $\mathrm{mm}$, ya que la pulverización también genera variación (Givens et al., 1997; Jiménez, 2007).

En cuanto al número de muestras que deben participar en el proceso de calibración y validación de los modelos de predicción desarrollados en el NIRS, no existe consenso sobre un número ideal, éste dependerá del objetivo del análisis como lo reportan varios grupos de investigación (Tabla 2). En forrajes es recomendado 50 muestras para el análisis del regresión del componente principal (Dryden, 2003). En entidades químicas simples de productos homogéneos puede bastar con 30 a 40 muestras, mientras si por el contrario se pretende evaluar el contenido de proteína de productos heterogéneos se requieren un mínimo de 100 muestras (Alomar, 1997). Las muestras deben incluir todas las posibles fuentes de variación del alimento a ser analizado no solo bajo las condiciones actuales sino para futuros análisis Entre ellas y para análisis de calidad de forrajes se consideran las siguientes variables: estación del año, localización, topografía, suelos, manejo de los potreros (Andueza-, Picard, Jestin, Andrieu, \& Baumont, 2011).
Tabla 2. Número de muestras sugeridas para calibración del NIRS de acuerdo al tipo de análisis de

\begin{tabular}{lc}
\multicolumn{1}{c}{ Tipo de análisis } & n \\
\hline Calidad nutricional de gramíneas & 237 \\
Planta de maíz & 40 a 60 \\
Calidad de dietas, perfil fecal & 148 \\
Proteína, FDA de heno & 650 \\
Grano de maíz & 262 \\
Digestibilidad in vitro de la MS de Lucerna & 166 \\
Digestibilidad in vitro de la MO de heno de & 35 por especie \\
pasto & \\
\hline Fuente: D’Mello, 2000 &
\end{tabular}

Varios métodos han sido desarrollados para la selección de las muestras de calibración: el primero, consiste en seleccionar muestras al azar; el segundo, es usar una muestras cada $n$ número de muestras, por ejemplo de 2000 muestras se realizan grupos de 20 muestras y de cada grupo se selecciona una muestra para calibración; el último, consiste en escanear todas las muestras y después seleccionarlas con base en su diferencia espectral. Elegir 100 muestras espectralmente diferentes es un número aceptable para comenzar la calibración. No obstante, dependiendo de la diversidad de las muestras puede ser que 100 muestras no sean representativas.

El objetivo de la calibración es encontrar la relación entre la información espectral y el análisis o la variable de interés (De Mello, 2000).

\section{Desarrollo de los modelos de prediccion}

Para el desarrollo de los modelos de predicción se han establecido varias etapas, las cuales son evaluadas de acuerdo a criterios estadísticos para conseguir modelos de predicción confiables de acuerdo a cada componente químico (Figura 2). 
1.Definir el área de muestreo

2.Colectar las muestras con la mayor variabilidad posible

3.Secar, moler y preparar las muestras

4.Tomar los espectros de las muestras

5.Seleccionar los espectros que representen la muestra

6. Realizar el análisis de laboratorio de las muestras seleccionadas usando el método de referencia

7.Confrontar los resultados del método de referencia con sus respectivos espectros para generar la ecuación de calibración

8.Seleccionar un grupo de muestras para validación interna y realizar el análisis por el método de referencia

9.Validar la ecuación de calibración con los resultados de referencia de las muestras de validación interna

10. Si los resultados del paso 9 son satisfactorios, utilizar la ecuación de calibración para análisis de rutina, si no los son repetir del paso $\underline{\mathbf{5} \text { al } \mathbf{9}}$

Figura 2. Elaboración propiaPasos sugeridos para la calibración NIRS.

Fuente: Adaptado de Dardenne en http://www.feedipedia.org/content/nirs-feed-and-soil-analysisdeveloping-countries Acceso: marzo 5 de 2015

Los datos de calibración son obtenidos del material que reúne la variación espectral y química, removiendo datos outlier del grupo de datos de calibración (Shenk y Westerhaus, 1991 citado por Jiménez, 2007). Las muestras destinadas a calibración, deben ser escaneadas de forma aleatoria para disminuir el error asociado a condiciones ambientales, como la temperatura y a las condiciones del equipo. Una vez son obtenidos los espectros en el equipo, se evalúan para determinar si existe alguno que difiera en los picos de absorción especialmente en la región 400-600nm y si la línea base es desplazada hacia arriba, asociado a la diferencia en el tamaño de las partículas de las muestras. Cuando el espectro NIR de un material dado tiende a ser similar, tales diferencias espectrales indican errores que deben ser ajustados (Tabla 3). La evaluación espectral es realizada por el software diseñado para tal fin y que está instalado junto con el equipo NIRS.

Varios factores pueden afectar la calibración del NIRS, por lo cual es necesario considerar la temperatura del ambiente, la temperatura de la muestra y el voltaje del equipo (Pojić, Mastilović, \& Majcen, 2012).

Tabla 3. Factores que generan errores en el desarrollo de modelos de predicción

\begin{tabular}{ll}
\hline Factor & Efecto Espectral \\
\hline Tamaño de la partícula & Cambios en la línea base \\
Temperatura & Picos en el espectro \\
Embalaje de la muestra & El espectro no representa la \\
& muestra \\
Muestra homogénea & Muestra no representativa \\
Componente fuera de rango & Calibración inadecuada \\
Diferentes alimentos o & Calibración inadecuada \\
muestras & \\
Reparación del espectroscopio & Cambios en los picos y/o \\
& absorbancia \\
\hline
\end{tabular}

Fuente: D’Mello, 2000

La metodología utilizada para el análisis de la información en la fase de calibración, es el análisis multivariado, específicamente la regresión de mínimos cuadrados parciales (PLS), el cual relaciona dos matrices X y Y, asociando las estructuras químicas presentes en la muestra con sus propiedades químicas. Este análisis es también el adecuado cuando el equipo NIRS es de reflectancia (Wold, Sjöström, \& Eriksson, 2001). El PLS busca los componentes principales que estén asociados para explicar la variación de $\mathrm{X}$ y predecir la respuesta en Y, por lo cual es necesario desarrollar un modelo de predicción distinto por cada componente químico de la misma muestra (Chemistry, n.d.; Givens et al., 1997).

Existen parámetros estadísticos que son utilizados para seleccionar las mejores ecuaciones o modelos de predicción por componente químico. Los párametros evaluados son el valor de error estandar de calibración y de validación cruzada, que debe ser el mas bajo posible o próximo a cero y el coeficiente de determinacion de calibracion y de validación, el cual debe ser lo más próximo a uno. Estos parametros son generados en cada proceso de calibración, 
después de que los espectros tomados son convertidos a valores numéricos para ser aplicados tratamientos matemáticos. Todos estos procesos son realizados en el software del equipo desarrollado para tal fin (“ANALYSIS OF FORAGE RESEARCH

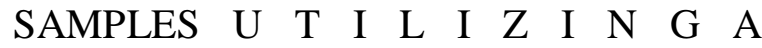
COMBINATION OF WET CHEMISTRY A N D NEAR IN FRARED R E FL ECTANCE SPECTROSCOPY 1," 1987; Buxton \& Mertens, 1991).

los modelos desarrollados en un equipo NIRS pueden ser transferidos para otro equipo, sin embargo para ello es necesario realizar la transeferencia de calibracion, aplicando modelos estadísticos adecuados para ellos, de lo contrario la predicción de un component químico desarrollado en un equipo deber ser siempre analizada en el mismo equipo (Honorato, Neto, Martins, Galvão, \& Pimentel, 2007).

\section{NIRS aplicado al análisis de alimentos utilizados en nutrición animal}

En alimentos destinados a la nutrición animal el NIRS puede ser aplicado para desarrollar modelos de predicción en gramineas y leguminosas, con variaciones en la predicción dependiendo del componente químico y de la especie forrajera. Esta metodología ha sido aplicada en varios países de Europa y en America, siendo en América Latina Brasil el país que está a la vanguardia de dicha tecnología.

Los componentes que han sido determinados con confiabilidad son: materia seca (MS), proteína, fibra en detergente neutra (FDN), fibra en detergente ácido (FDA). En ensilajes destinados a la alimentación de bovinos han sido determinados los mismos componentes con precisión en la estimativa de los componentes nutricionales (de Boever, Cottyn, Vanacker, \& Boucqué, 1995; De Boever, Cottyn, De Brabander, Vanacker, \& Boucqué, 1996; Stuth, Jama, \& Tolleson, 2003; Ohlsson, Houmøller,
Weisbjerg, Lund, \& Hvelplund, 2007). Para la determinación de carbohidratos no estructurales de forrajes tropicales (Brown, Piacitelli, \& Mislevy, 1987).

También existen reportes que la metodología puede ser aplicada para predecir la digestibilidad de la materia orgánica y la energía neta y metabolizable de plantas de cereales, para lo cual se requiere un periodo de colecta mínimo de 4 años y que hayan tenido diferentes protocolos de fertilización (Bruno-Soares, Murray, Paterson, \& Abreu, 1998; Volkers, Wachendorf, Loges, Jovanovic, \& Taube, 2003; Deaville, Humphries, \& Givens, 2009 ). Ha sido además utilizado en la predicción de minerales de leguminosas, tales como B, $\mathrm{Cu}, \mathrm{Mn}, \mathrm{Zn}, \mathrm{Na}$ y $\mathrm{S}$ aunque la precisión es menor comparada con las metodologías convencionales (Cozzolino \& Moron, 2004). En ovinos en pastoreo ha permitido la predicción de la digestibilidad de la materia orgánica y de la proteína del forraje consumido (Cox, Vasconselos, \& Leite, 2000). En leguminosas como la alfalfa y la leucaena, para estimar contenidos de PB, FDN y la digestibilidad de la MS, así como en la Leucanea fueron estimados componentes reconocidos comúnmente como factores antinutricionales, tales como la mimosina, fenoles y taninos (Wheeler, Chaney, Johnson, \& Butler, n.d. ;Prasad, 1995; De Boever et al., 1996; Brogna et al., 2009).

En silo de maíz ha sido utilizado para la evaluación de MS, proteína, FDN y FDA con coeficientes de determinación de validación superiores a 0.98 para todos los componentes (Fontaneli, Durr, SchefferBasso, Haubert, \& Bortolini, 2002). Asi mismo, ha sido usado para predecir el valor energético de alimentos para dietas de porcinos (Aufrère, Graviou, Demarquilly, Perez, \& Andrieu, 1996). En gramíneas, ha sido aplicado como una herramienta para conocer el valor nutricional de gramíneas 
Cynodon spp, utilizadas en la alimentación de ganado de leche en el sur de Brasil, estimando componentes fibrosos, proteína y minerales con valores más confiables para los componentes orgánicos que para los inorgánicos (Fontaneli et al., 2004). En el caso de potreros con mezcla de gramíneas has sido posible determinar la composición de Dactylis glomerata L., Lolium perenne, Festuca arundinacea Schreb) y Trifolium repens, siendo superior en la predicción de este ultimo (Surault, Briand, Veron, \& Huyghe, 2005). En la determinación de la tasa de degradación de la FDN de gramíneas $\mathrm{C}_{3}$ y de leguminosas tales como Phleum pratense, Festuca pratensis, Dactylis glomerata, Bromus inermis, Trifolium pratense, (Nordheim, Volden, Fystro, \& Lunnan, 2007). También se ha reconocido el potencial que tiene la metodología para estimar la digestibilidad de forrajes y en el análisis de heces de bovinos en pastoreo por lo cual permitirá mejorar el desempeño en pastoreo (Decruyenaere, Planchon, Dardenne, \& Stilmant, 2015).

Otra aplicación del NIRS, además de determinar composición química en forrajes es la de predecir la producción de gases de especies forrajeras, inclusive con mayor precisión que las metodologías tradicionalmente utilizadas, debido a que el NIRS asocia la información de los componentes químicos de la planta con las propiedades físicas de la muestra. (Andrés et al., 2005).

En Colombia, ha sido utilizada para análisis de caña de azúcar y en gramíneas como el Pennisetum clandestinum según estudio realizado por A.Rivera en la Universidad de Antioquia y Universidad Nacional de Colombia, con resultados favorables para la predicción de PB, FDN y FDA (Meeting \& City, 2016).

Por las anteriores razones, en forrajes y/o alimentos destinados a nutrición animal, ha sido reconocida como una técnica de bajo costo, con resultados confiables, y que sirve de herramienta para el balanceo de raciones o la formulación de un suplemento (Corson, Waghorn, Ulyatt, \& Lee, 1999). Siendo importante resaltar que las muestras colectadas para el desarrollo de los modelos de predicción deben considerar todos los factores de variación ambiental, de suelos y de manejo para que la ecuación sea capaz de predecir una muestras procedente de diferentes regiones (Cougnon, Waes, Dardenne, Baert, \& Reheul, 2013).

En la industria de alimentos ha sido utilizado en la evaluación de la calidad de frutas y vegetales, siendo importante contar con un modelo con gran cantidad de muestras para predecir materia seca, acidez, textura y alteraciones propias de los vegetales (Nicolaï et al., 2007; Inácio, De Lima, Lopes, Pessoa, \& De Almeida Teixeira, 2013).

Otros usos diferentes del NIRS diferentes a la evaluación de alimentos es la industria maderable, esta ha sido útil en la predicción de parámetros de calidad de madera como el Eucaliptus, ya que permite el análisis de lignina (de Sousa, Gomide, Milagres, \& de Almeida, 2011). De otra parte, es una alternativa no tradicional para verificar la autenticidad de productos detectando alimentos adulterados (Rodriguez-Saona \& Allendorf, 2011; De Souza \& Poppi, 2012). En África se ha usado para predecir la fertilidad del suelo con resultados satisfactorios (Selmet \& Bimont, 2014).

Como consideraciones finales, es relevante destacar que en forrajes y alimentos usados en nutrición animal, autores afirman que para algunos componentes químicos el NIRS es capaz de realizar estimativas más precisas y exactas que por métodos convencionales, esto debido al principio quimiométrico que aplica la técnica (Albrecht, Marten, Halgerson, \& Wedin, 1987). Siendo una técnica relativamente nueva comparada con métodos 
convencionales tiene futuro debido a la reducción de costos, tiempo y que no genera desechos químicos.

\section{CONCLUSIONES}

El NIRS es una metodología que relaciona la composición química de una materia con la absorción de luz del segmento infrarrojo cercano del espectro electromagnético.

Los modelos de predicción desarrollados en el NIRS para componentes químicos de los alimentos y de otros materiales son confiables y precisos.

El NIRS es un método de laboratorio limpio, rápido y de bajo costo para análisis de alimentos usados en nutrición animal.

\section{REFERENCIAS BIBLIOGRÁFICAS}

Alomar, D., \& Fuhslocher, R. (1997). Fundamentos De La Espectroscopia De Reflectancia En El Infrarojo Cercano (Nirs) Como Método De Análisis De Forrajes, 5. Retrieved from http://mingaonline.uach.cl/scielo.php?pid=S $\underline{0304-}$ $\underline{88021998000100011 \& \text { script=sci_arttext }}$

Albrecht, K. A., Marten, G. C., Halgerson, J. L., \& Wedin, W. F. (1987). Analysis of Cell-Wall Carbohydrates and Starch in Alfalfa, 588, 586-588. ANALYSIS OF FORAGE RESEARCH SAMPLES U T I L I Z I N G A COMBINATION OF WET CHEMISTRY A N D NEAR IN FRARED R E FL ECTANCE SPECTROSCOPY 1. (1987), 271-282.

Andrés, S., Calleja, A., López, S., González, J. S., Rodríguez, P. L., \& Giráldez, F. J. (2005). Prediction of gas production kinetic parameters of forages by chemical composition and near infrared reflectance spectroscopy. Animal Feed Science and Technology, 123-124 Pa, 487-499. https://doi.org/10.1016/j.anifeedsci.2005.04. 043

Andueza-, D., Picard, F., Jestin, M., Andrieu, J., \& Baumont, R. (2011). NIRS prediction of the feed value of temperate forages: efficacy of four calibration strategies. Animal, 5(7), 1002-1013. https://doi.org/10.1017/S175173111000269 7

Aufrère, J., Graviou, D., Demarquilly, C., Perez, J. M., \& Andrieu, J. (1996). Near infrared reflectance spectroscopy to predict energy value of compound feeds for swine and ruminants. Animal Feed Science and Technology, 62(2-4), 77-90. https://doi.org/http://dx.doi.org/10.1016/S03 77-8401(96)00995-9

Brogna, N., Pacchioli, M. T., Immovilli, A., Ruozzi, F., Ward, R., \& Formigoni, A. (2009). The use of near-infrared reflectance spectroscopy (NIRS) in the prediction of chemical composition and in vitro neutral detergent fiber (NDF) digestibility of Italian alfalfa hay. Italian Journal of Animal Science, 8(SUPPL. 2), 271-273. https://doi.org/10.4081/ijas.2009.s2.271

Brown, W. F., Piacitelli, C. K., \& Mislevy, P. (1987). Near infrared reflectance analysis of nonstructural carbohydrate concentration in tropical grasses. Crop Science, 27, 786788.

Bruno-Soares, A. M., Murray, I., Paterson, R. M., \& Abreu, J. M. F. F. (1998). Use of near infrared reflectance spectroscopy (NIRS) for the prediction of the chemical composition and nutritional attributes of green crop cereals. Animal Feed Science and Technology, 75(1), 15-25. https://doi.org/10.1016/S0377- 


\section{1(98)00190-4}

Buxton, D. R., \& Mertens, D. R. (1991). Errors in forage-quality data predicted by near infrared reflectance spectroscopy. Crop Science, 31(1), 212-218.

Cao, N. (2013). Calibration optimization and efficiency in near infrared spectroscopy, 183. Retrieved from http://lib.dr.iastate.edu/etd/13199/

Cozzolino, D. (2005). Uso De La Espectroscopía De Reflectancia En El Infrarrojo Cercano (Nirs) En El Analisis De Alimentos Para Animales. Agrociencia. VI, $25-32$.

Chemistry, F. (n.d.). No Title.

Corson, D. C., Waghorn, G. C., Ulyatt, M. J., \& Lee, J. (1999). NIRS : Forage analysis and livestock feeding. Proceedings of New Zealand Grassland Association, 61, 127132. Retrieved from http://www.grassland.org.nz/publications/nz grassland_publication_507.pdf

Cougnon, M., Waes, C. Van, Dardenne, P., Baert, J., \& Reheul, D. (2013). Grass and Forage Science Comparison of near infrared reflectance spectroscopy calibration strategies for the botanical composition of grass-clover mixtures, (October 2012), 167175. https://doi.org/10.1111/gfs.12031

Cox, M., Vasconselos, V. R., \& Leite, E. R. (2000). Utilizacuo da Tecnica da Espectroscopia e Reflectlincia do InfraVermelho Proximo (NIRS) na Determinacuo do Valor Nutricional da Dieta de Ovinos em Caatinga. Revista Científica de Produção Animal, 2(1), 31-40.

Cozzolino, D., \& Moron, A. (2004). Exploring the use of near infrared reflectance spectroscopy (NIRS) to predict trace minerals in legumes. Animal Feed Science and Technology, 111(1-4), 161173.

https://doi.org/10.1016/j.anifeedsci.2003.08. 001

D’Mello, J. P. F. (2000). Farm animal metabolism and nutrition., 438. https://doi.org/10.1079/9780851993782.000 0

De Boever, J. L., Cottyn, B. G., De Brabander, D. L., Vanacker, J. M., \& Boucqué, C. V. (1996). Prediction of the feeding value of grass silages by chemical parameters, in vitro digestibility and nearinfrared reflectance spectroscopy. Animal Feed Science and Technology, 60(1-2), 103-115. $\quad$ https://doi.org/10.1016/03778401(95)00914-0

de Boever, J. L., Cottyn, B. G., Vanacker, J. M., \& Boucqué, C. V. (1995). The use of NIRS to predict the chemical composition and the energy value of compound feeds for cattle. Animal Feed Science and Technology, 51(3-4), 243-253. https://doi.org/10.1016/0377-

8401(94)00695-6

de Sousa, L. C., Gomide, J. L., Milagres, F. R., \& de Almeida, D. P. (2011). Desenvolvimento de modelos de calibra????o NIRS para minimiza????o das an??lises de madeiras de eucalyptus spp. Ciencia Florestal, 21(3), 589-597.

De Souza, A. M., \& Poppi, R. J. (2012). Experimento didático de quimiometria para análise exploratória de óleos vegetais comestíveis por espectroscopia no infravermelho médio e análise de componentes principais: UM tutorial, parte I. Quimica Nova, 35(1), 223-229. https://doi.org/10.1590/S0100- 


\section{9}

Deaville, E. R., Humphries, D. J., \& Givens, D. I. (2009). Whole crop cereals. 2. Prediction of apparent digestibility and energy value from in vitro digestion techniques and near infrared reflectance spectroscopy and of chemical composition by near infrared reflectance spectroscopy. Animal Feed Science and Technology, 149(1-2), 114-124. https://doi.org/10.1016/j.anifeedsci.2008.05. 008

Decruyenaere, V., Planchon, V., Dardenne, P., \& Stilmant, D. (2015). Prediction error and repeatability of near infrared reflectance spectroscopy applied to faeces samples in order to predict voluntary intake and digestibility of forages by ruminants. Animal Feed Science and Technology, 205, 49-59. https://doi.org/10.1016/j.anifeedsci.2015.04. 011

Fontaneli, R. S., Durr, J. W., SchefferBasso, S. M., Haubert, F., \& Bortolini, F. (2002). Validação do Método da Reflectância no Infravermelho Proximal para Análise de Silagem de Milho. Revista Brasileira de Zootecnia, 31, 594-598. https://doi.org/10.1590/S151635982002000300008

Fontaneli, R. S., Scheffer-Basso, S. M., Dürr, J. W., Appelt, J. V., Bortolini, F., \& Haubert, F. A. (2004). Predição da composição química de bermudas (Cynodon spp.) pela espectroscopia de reflectância no infravermelho proximal. Revista Brasileira de Zootecnia, 33(4), 838-842.

Honorato, F. A., Neto, B. D. B., Martins, M. N., Galvão, R. K. H., \& Pimentel, M. F. (2007). Transferência de calibração em métodos multivariados. Quimica Nova, 30(5),
https://doi.org/10.1590/S0100-

40422007000500044

Inácio, M. R. C., De Lima, K. M. G., Lopes, V. G., Pessoa, J. D. C., \& De Almeida Teixeira, G. H. (2013). Total anthocyanin content determination in intact a??a?? (Euterpe oleracea Mart.) and palmiteroju??ara (Euterpe edulis Mart.) fruit using near infrared spectroscopy (NIR) and multivariate calibration. Food Chemistry, 136(3-4), 1160-1164. https://doi.org/10.1016/j.foodchem.2012.09. 046

Dryden, G. M. (2003). Near Infrared Reflectance Spectroscopy: Applications in Deer Nutrition 47 341, (July).

Givens, D. I., DeBoever, J. L., \& Deaville, E. R. (1997). The principles, practices and some future applications of near infrared spectroscopy for predicting the nutritive value of foods for animals and humans 162 162. Nutrition Research Reviews, 10, 83114. Retrieved from wos: A1997YK89600004

Herrero, M., Murray, I., Fawcett, R. H., \& Dent, J. B. (1996). Prediction of the in vitro gas production and chemical composition of kikuyu grass by near-infrared reflectance spectroscopy. Animal Feed Science and Technology, 60, 51-67. https://doi.org/10.1016/0377$\underline{8401(95) 00924-8}$

Jiménez Paola Andrea, T. (2007). Identificación de harinas de yuca (Manihot esculenta crantz) con alto contenido proteico mediante espectroscopia de infrarrojo cercano (NIRS). Producción Animal Tropical, MAESTRIA E, 71.

Lascano, C. E. (2002). Caracterización de las pasturas para maximizar producción 
animal Characterization of pastures to maximize animal production Recibido Marzo 15, 2001Aceptado Enero 10 , 2002 Conferencia invitada presentada en la XVI Reunión de la Asociación Latinoameric. Archivos Latinoamericanos de Producción Animal, 10, 126-132.

Pasos sugeridos para la calibración NIRS. (Adaptado de Dardenne en: http://www.feedipedia.org/content/nirs-feedand-soil-analysis-developing-countries

Acceso: marzo 5 de 2015).

Radiación infrarroja. (a) Reflexión; (b) reflexión difusa; (c) absorción; (d) transmisión; (e) difracción; (f) difusión (Givens et al., 1997; En: http://www.engormix.com/MAbalanceados/formulacion/articulos/espectros copia- infrarrojo-cercano-nirs-t577/800p0.htm, Acceso:marzo de 2015).

Sandoval-mej, L. A., \& Bueso-ucl, F. J. (2008). Predicción Nutricional Para Pastos Tropicales Por. Agronomía Mesoamericana, 19(2), 221225. https://doi.org/1021-7444

Vásquez, D. R., Abadía, B., \& Arreaza, L. C. (2004). Aplicación de la Espectroscopía de Reflectancia en el Infrarrojo Cercano (NIRS) para la caracterización nutricional del pasto Guinea y del grano de maíz. Revista Corpoica, 5(Octubre), 49-55.

Landau, S., Glasser, T., \& Dvash, L. (2006). Monitoring nutrition in small ruminants with the aid of near infrared reflectance spectroscopy (NIRS) technology: A review. Small Ruminant Research, 61(1), 1-11. https://doi.org/10.1016/j.smallrumres.2004.1 2.012

Meeting, J. A., \& City, S. L. (2016). 2016 Jam, 94.

Nicolaï, B. M., Beullens, K., Bobelyn, E., Peirs, A., Saeys, W., Theron, K. I., \& Lammertyn, J. (2007). Nondestructive measurement of fruit and vegetable quality by means of NIR spectroscopy: A review. Postharvest Biology and Technology, 46(2), 99-118.

https://doi.org/10.1016/j.postharvbio.2007.0 6.024

Nordheim, H., Volden, H., Fystro, G., \& Lunnan, T. (2007). Prediction of in situ degradation characteristics of neutral detergent fibre (aNDF) in temperate grasses and red clover using near-infrared reflectance spectroscopy (NIRS). Animal Feed Science and Technology, 139(1-2), 92-108.

https://doi.org/10.1016/j.anifeedsci.2006.11. 024

Ohlsson, C., Houmøller, L. P., Weisbjerg, M. R., Lund, P., \& Hvelplund, T. (2007). Effective rumen degradation of dry matter, crude protein and neutral detergent fibre in forage determined by near infrared reflectance spectroscopy. Journal of Animal Physiology and Animal Nutrition, 91(1112), 498-507.

https://doi.org/10.1111/j.1439-

0396.2007.00683.x

Pasquini, C. (2003). Near infrared spectroscopy: Fundamentals, practical aspects and analytical applications. Journal of the Brazilian Chemical Society, 14(2), 198-219. https://doi.org/10.1590/S010350532003000200006

Pojić, M., Mastilović, J., \& Majcen, N. (2012). Robustness of the near infrared spectroscopy method determined using univariate and multivariate approach. Food Chemistry, 134(3), 1699-1705. https://doi.org/10.1016/j.foodchem.2012.03. 104

Prasad, M. N. V. (1995). Analysis of Leucaena mimosine, Acacia tannins and 
total phenols by near infrared reflectance spectroscopy. Biomass and Bioenergy, 8(3), 203-205. https://doi.org/10.1016/09619534(95)00010-5

Rodriguez-Saona, L. E., \& Allendorf, M. E. (2011). Use of FTIR for Rapid Authentication and Detection of Adulteration of Food. Annual Review of Food Science and Technology, 2(1), 467483. https://doi.org/10.1146/annurev-food022510-133750

Selmet, C. U. M. R., \& Bimont, P. (2014). The use of Near-Infrared Spectrometry ( NIRS ) to predict the soil fertility in South Africa, 2013-2014.

Stuth, J., Jama, A., \& Tolleson, D. (2003). Direct and indirect means of predicting forage quality through near infrared reflectance spectroscopy. Field Crops Research, 84(1-2), 45-56. https://doi.org/10.1016/S03784290(03)00140-0

Surault, F., Briand, M., Veron, R., \& Huyghe, C. (2005). NIRS calibration equations to determine species contribution in grassland swards, 11, 2004-2006.

Volkers, K. C., Wachendorf, M., Loges, R., Jovanovic, N. J., \& Taube, F. (2003). Prediction of the quality of forage maize by near-infrared reflectance spectroscopy. Animal Feed Science and Technology, 109(1-4), 183-194. chemometrics. Chemometrics and Intelligent Laboratory Systems, 58(2), 109-130. https://doi.org/10.1016/S01697439(01)00155-1

\section{FINANCIAMIENTO}

Agradecimientos a Colciencias 656 de 2014 en la Convocatoria "Es Tiempo de Volver" y a la Universidad Nacional de Colombia en la estancia posdoctoral

https://doi.org/10.1016/S0377-

8401(03)00173-1

Wheeler, R. A., Chaney, W. R., Johnson, K. D., \& Butler, L. G. (n.d.). Leucaena forage analysis using near infrared reflectance spectroscopy, 64(3 17).

Wold, S., Sjöström, M., \& Eriksson, L. (2001). PLS-regression: A basic tool of 